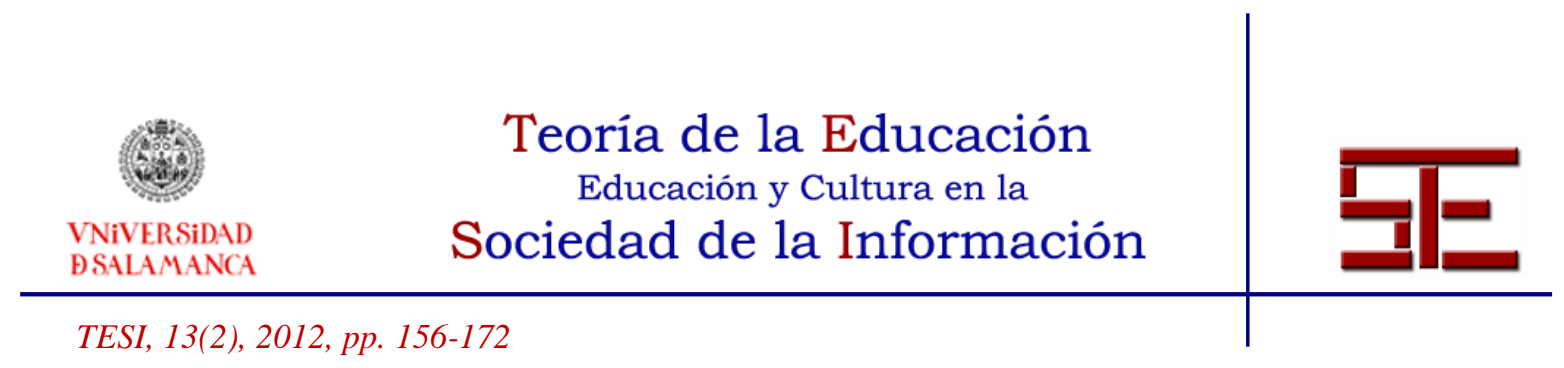

\title{
PROPUESTA DOCENTE PARA LAS PRÁCTICAS DE ROBÓTICA EN EL GRADO DE INGENIERÍA INFORMÁTICA
}

Resumen: En este artículo se realiza una propuesta para la docencia de la parte práctica de una asignatura de robótica en una titulación de Grado en Ingeniería Informática. Con objeto de que la asignatura sea atractiva y fomentar el interés por parte del alumnado se ha huido de descripciones matemáticas complejas buscado aspectos más comunes a los estudiantes de la titulación como la robótica móvil. Para que el alumnado tenga acceso a practicar con un robot real se ha elegido un robot comercial de bajo coste como es Roomba.

La metodología docente se basa en la elaboración de proyectos tras el visionado de unos videos del robot realizando una acción para que estos consigan reproducirla lo mejor posible, de modo que puedan extraer sus propias conclusiones para llevarlas a la práctica. Así mismo se presentan el conjunto de proyectos que deberán realizar los estudiantes.

Palabras clave: Robótica; Roomba; Grado; Informática

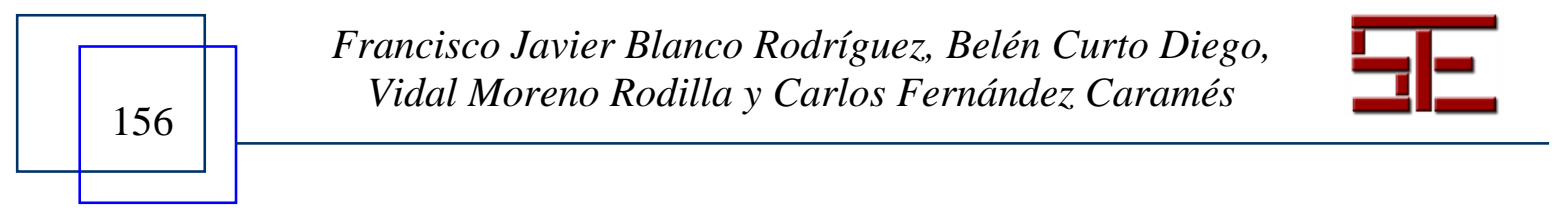




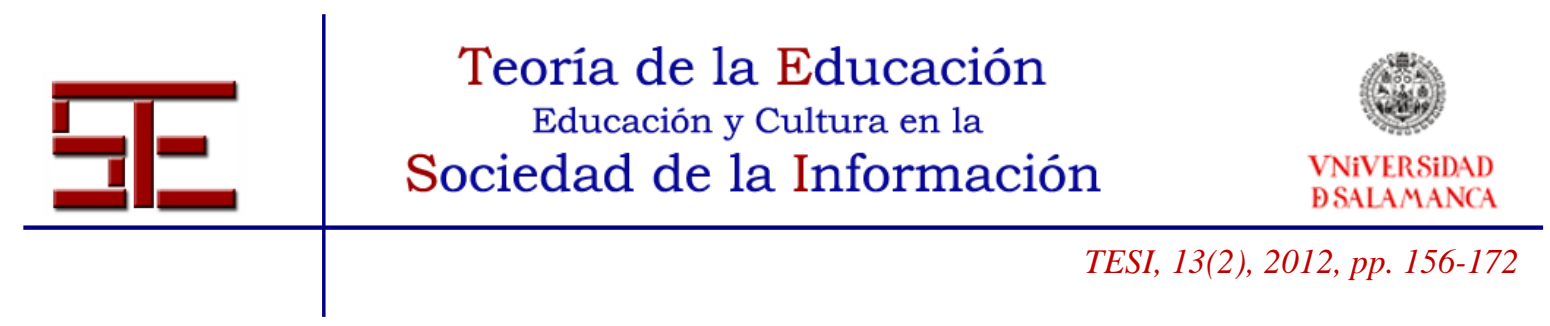

\section{ACADEMIC PROPOSAL TO PRACTICAL TEACHING OF ROBOTICS IN COMPUTER SCIENCE GRADE}

Abstract: In this paper we present a proposal for the practical teaching robotics in a Degree in Computer Science. In order to make the course more attractive and stimulate the interest by students complex mathematical descriptions had been avoided. Instead, it focuses in other aspects more related to those degree students such as mobile robotics. To allow students to have access to real robot to practice Roomba robot has been chosen.

Teaching methodology is based on project development by students after viewing of a set of videos of the robot performing actions. Then students must obtain a program where the robot behaves in the same way. So, they first have to analyze how the robot gets the behavior to implementing it later. It also presents the set of projects to be undertaken by students.

Keywords: Robotics; Roomba; Graduate; Computer Science.

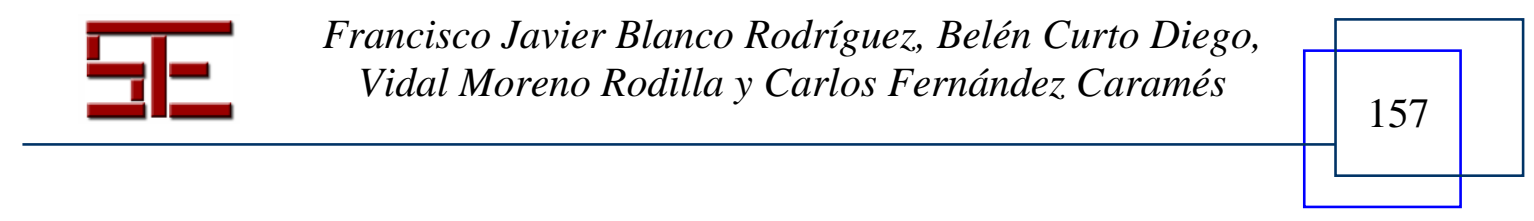




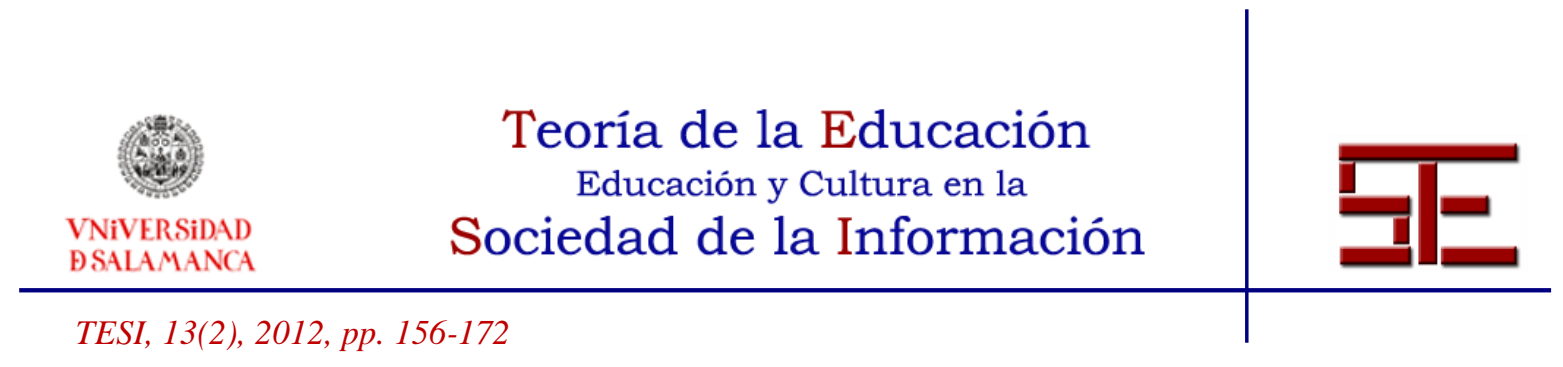

\title{
PROPUESTA DOCENTE PARA LAS PRÁCTICAS DE ROBÓTICA EN EL GRADO DE INGENIERÍA INFORMÁTICA
}

\author{
Fecha de recepción: 31/05/2012; fecha de aceptación: 13/07/2012; fecha de publicación: 26/07/2012
}

Francisco Javier Blanco Rodríguez

fjblanco@usal.es

Universidad de Salamanca

Belén Curto Diego

bcurto@usal.es

Universidad de Salamanca

Vidal Moreno Rodilla

vmoreno@usal.es

Universidad de Salamanca

Carlos Fernández Caramés

carlosfc@usal.es

Universidad de Salamanca

\section{1.- INTRODUCCIÓN}

Cuando se han de considerar las competencias que se deben alcanzar en la planificación de una asignatura de Robótica general es necesario tener presentes los avances que se han producido en las últimas décadas, relacionados con los robots autónomos y que despiertan un gran interés en la sociedad y, por tanto, en el alumnado. Si además la asignatura está orientada a alumnos con una sólida base en programación, como será en el caso del Grado en Ingeniería Informática, ese interés se puede trasladar a un escenario práctico que les permita utilizar todas las habilidades adquiridas en cursos anteriores [13][4]. De este modo, se puede alcanzar un mayor grado de motivación e interés por la materia.

De este modo, el planteamiento de la materia será hacia la robótica móvil y alejarse de descripciones matemáticas complejas que pueden resultar un obstáculo a estudiantes de esta titulación.

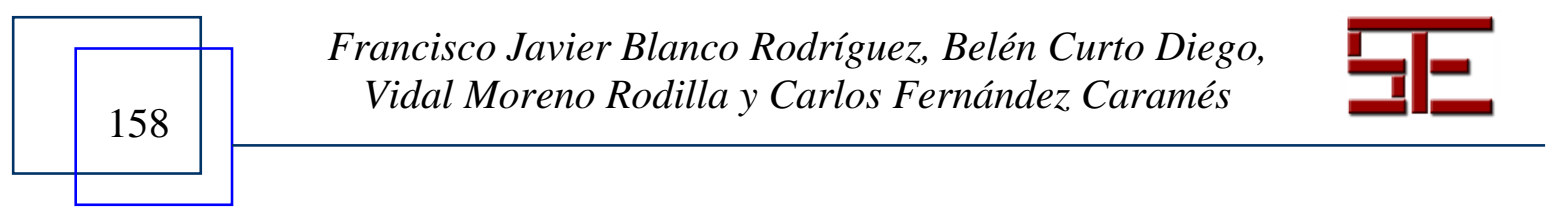




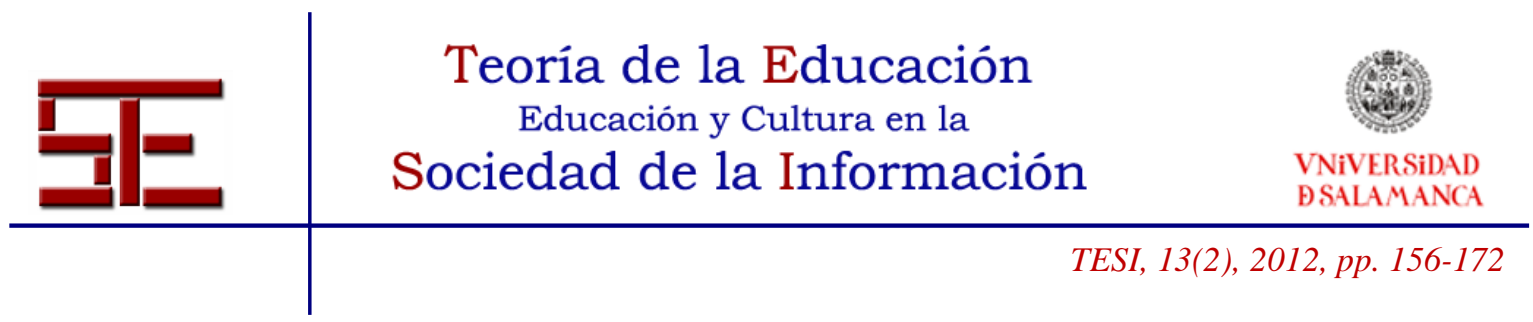

Por otro lado, es necesario también establecer cuáles son los recursos que se deben utilizar para el desarrollo de las sesiones prácticas. Debemos considerar en primer lugar la utilización de simuladores. Existen gran variedad de ellos, tanto comerciales como de uso libre, que incluyen gran realismo con gráficos $3 \mathrm{D}$, control de colisiones, cámaras de video virtuales, etc. Pero el uso de simuladores, aunque muy útiles en gran cantidad de situaciones, puede alejar al alumno de la realidad sin ser consciente de la complejidad de trabajar con un dispositivo físico real.

Si nos centramos en la utilización de hardware, existe una amplia variedad de alternativas. Una de las primeras que podríamos considerar es la utilización de microrobots de construcción propia, basados en microcontroladores u otros procesadores [6].

El uso de estos micro-robots puede frenar a ciertos alumnos de una titulación de informática acostumbrados a programación en alto nivel y escasa experiencia en el manejo del hardware. Otra posibilidad es la utilización de los kits de Lego Mindstorms, los cuales disponen de gran variedad de sensores y una gran versatilidad para diseñar nuestro propio robot [11][12]. Aunque inicialmente estaban planteados para su utilización por niños, desde diversos entornos se han desarrollado sistemas (NQC o LeJOS) que permiten la realización de programas de alto nivel, con leguajes de programación habituales. Sin embargo, los robots que se pueden crear no tienen una utilización directa en tareas del mundo real, lo cual puede conducir a una falta de interés del alumnado.

Otra alternativa adicional es el uso de otros robots comerciales destinados a la docencia y la investigación, como pueden ser Pioneer, Garcia o Khepera. Sin embargo, su elevado coste de adquisición y mantenimiento hace que sea inviable disponer de un número suficiente de ellos para formar grupos de trabajo pequeños en las sesiones prácticas.

En un punto intermedio se encuentra el robot Roomba, un robot comercial creado por iRobot destinado a la limpieza. Este punto lo hace interesante puesto que es un claro ejemplo en el que la Robótica ha penetrado en nuestra vida cotidiana, lo que supone una forma de atraer la atención y aumentar la motivación de los estudiantes. Además, el coste es sensiblemente inferior si se compara con los robots comerciales construidos expresamente para docencia. Estas características hacen que se esté expandiendo el uso del robot Roomba en la docencia [5][14]. Otro robot con características muy similares y también desarrollado por iRobot es Create, enfocado concretamente a la docencia e

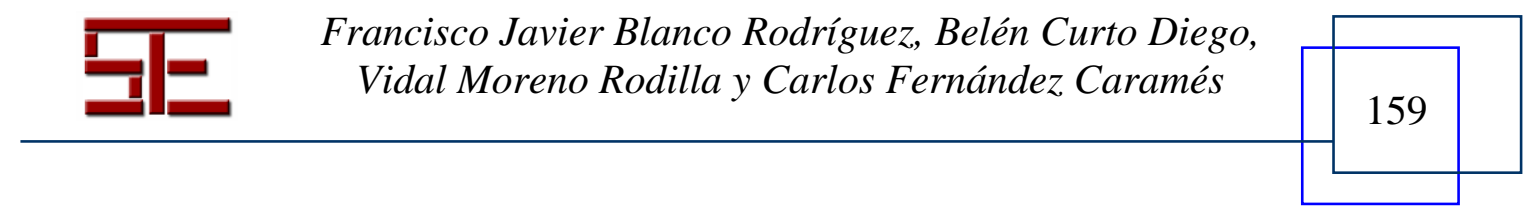




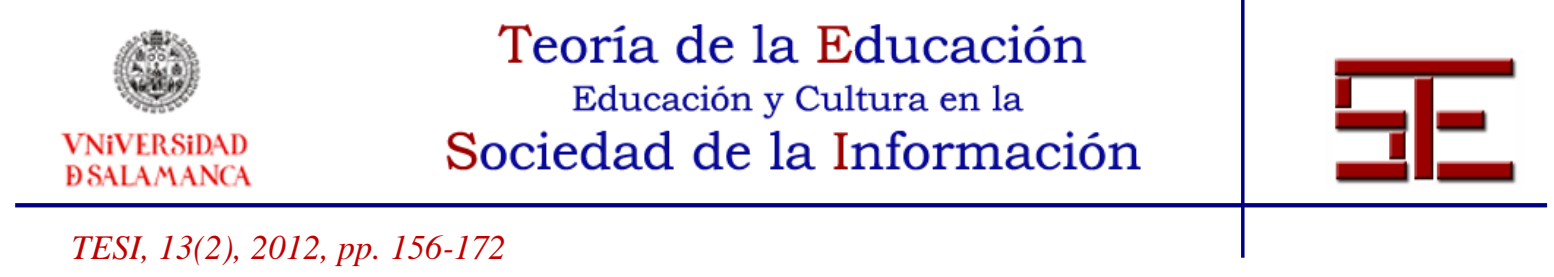

investigación y ampliamente utilizado [8]. No obstante este último robot no puede ser importado actualmente en España.

En la siguiente sección se expondrá el contexto académico donde se desarrolla la asignatura objeto de esta propuesta de prácticas. En la sección 3, se aborda la primera sesión práctica con una descripción del robot Roomba. Posteriormente, en la sección 4, se plantea la API para la programación del robot que se utilizará en las sesiones prácticas. En la sección 5 se describen las tareas propuestas para la realización por los estudiantes en sesiones no guiadas. Por último, se finaliza con las conclusiones.

\section{2.- CONTEXTO ACADÉMICO}

La propuesta que se realiza en este artículo se sitúa en la asignatura "Robótica" que se impartirá en el Grado de Ingeniería Informática en la Universidad de Salamanca. Ésta tiene un carácter optativo con una carga de 3 ECTS teóricos y 3 prácticos. Su carácter optativo es un elemento fundamental a tener en cuenta a la hora de elaborar un proyecto docente para la materia. También es necesario considerar que no existe otra asignatura relacionada con la Robótica en los planes de estudios de titulación, lo que condiciona claramente los contenidos y objetivos de la materia.

El entusiasmo que puede despertar en este alumnado este tipo de tecnología puede verse diluido ante un importante recelo a encontrarse con complicadas fórmulas matemáticas. Así, esta asignatura se ha enfocado hacia robots autónomos, hardware involucrado, arquitecturas software de control de robots y realizar las descripciones someras de la cinemática y la dinámica.

De este modo, el enfoque que se ha dado a la asignatura tiene dos vertientes: por un lado dar a conocer los principios y conceptos básicos de la robótica, y por el otro proporcionar una visión actual de la robótica autónoma. Por tanto, los objetivos son que el alumno sea capaz de:

- Conocer la evolución de la robótica hasta la actualidad

- Diferenciar los componentes que forman un robot y su estructura

- Identificar los diferentes tipos de sensores y actuadores

- Comprender la descripción matemática de robots móviles

- Conocer las técnicas básicas para el control a bajo nivel

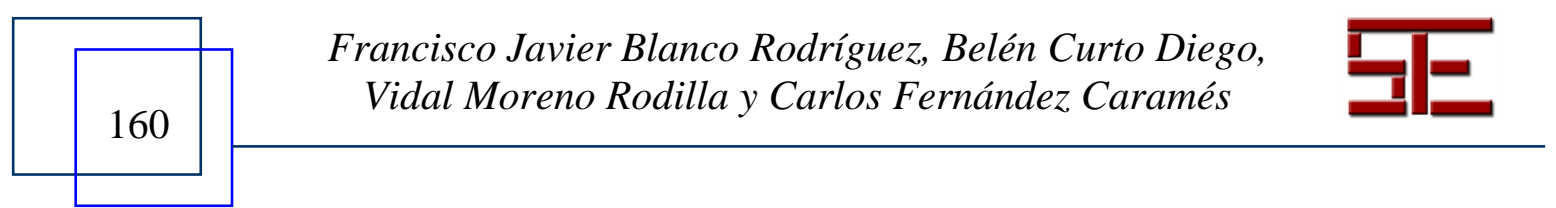




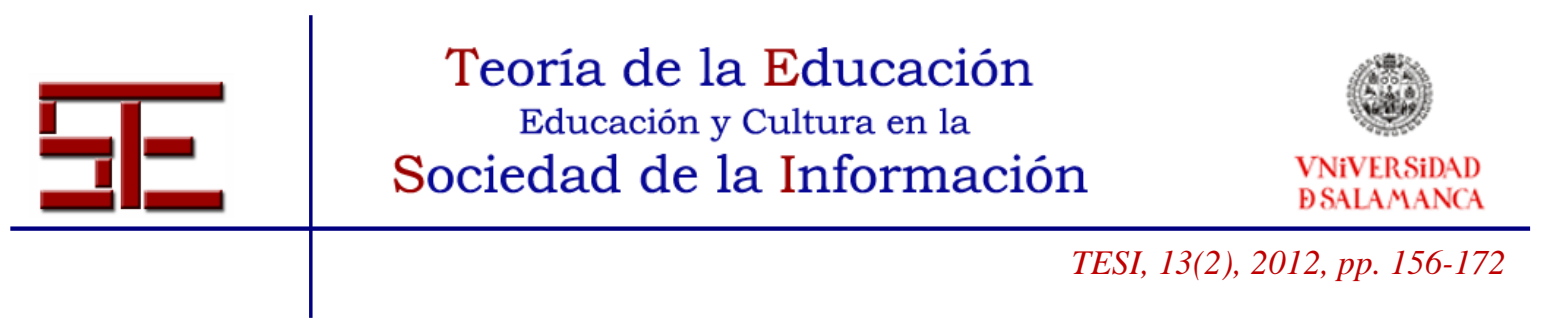

- Diferenciar entre los diferentes paradigmas de control a alto nivel

- Reconocer los elementos tanto de las arquitecturas reactivas como las deliberativas

- Darse cuenta de las principales dificultades que presenta la robótica autónoma

Por último, destacar que al tratarse de una materia optativa se pretende que tenga un carácter aplicado y práctico. Por esta razón, en las prácticas se procurará que puedan experimentar con los diferentes conceptos introducidos en las clases teóricas. Así, la propuesta de prácticas que se presenta en este artículo aborda los objetivos relacionados con el control a alto nivel y robots autónomos.

\section{3.- EL ROBOT ROOMBA}

Tal y como se comentó en la introducción se ha elegido el robot Roomba (Figura 1) como plataforma para la realización de las prácticas. Éste es comercializado por la empresa iRobot dentro de su gama de robots de limpieza. Desde el primer desarrollo en 2002 hasta la actualidad han aparecido diferentes versiones. Es necesario destacar que como se comentó previamente, desde hace ya algunos años, dado el uso que se ha venido dado a esta plataforma en diferentes universidades, iRobot ha desarrollado también una plataforma denominada iRobot Create destinada a investigación y docencia en el que se ha eliminado el hardware destinado a la limpieza y añadido un módulo para una programación más sencilla. Pero no se propone la utilización de esta plataforma puesto que no puede ser adquirida en España ni importada.

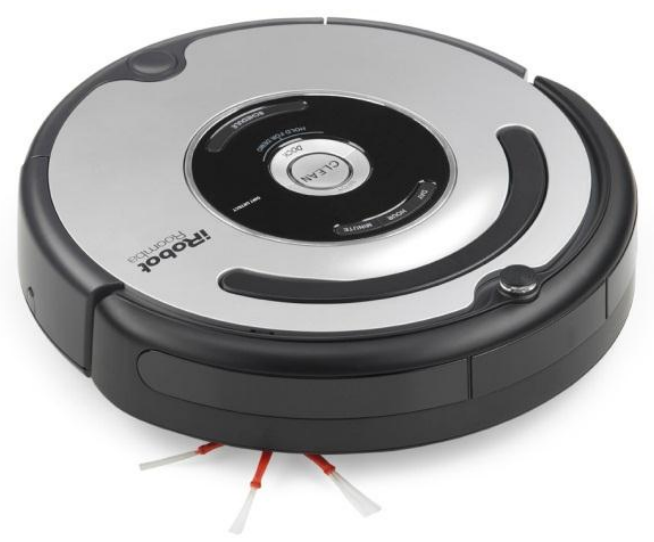

Figura 1: Robot Roomba modelo 555.

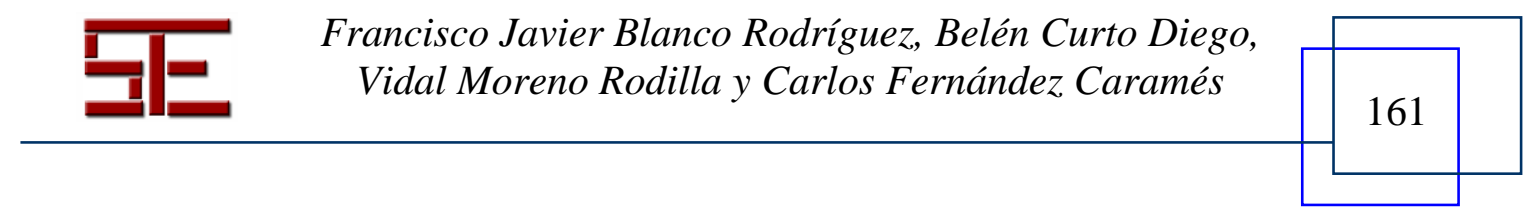




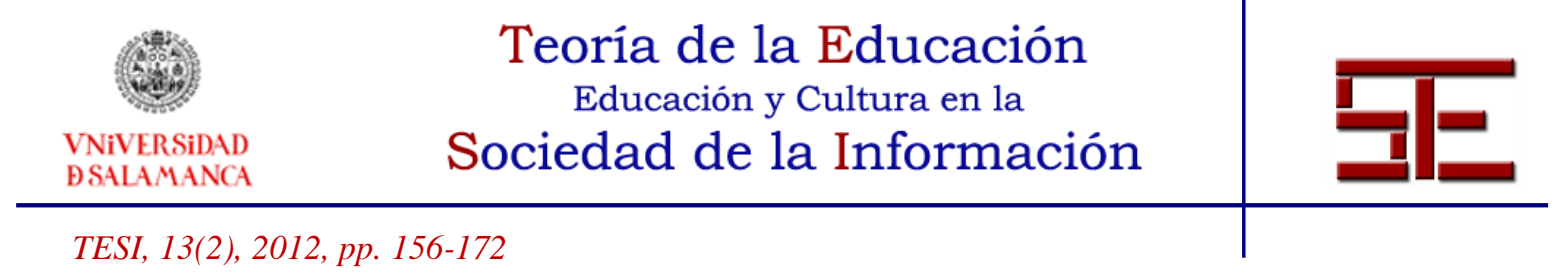

\section{1.- HARDWARE}

Para que su capacidad de movimientos sea amplia acorde a su tarea de limpieza su cinemática viene dada por una configuración diferencial, por lo que dispone de 2 motores independientes actuando sobre sus ruedas motrices. Adicionalmente cuenta con otros motores para accionar los cepillos para la limpieza.

Por otro lado dispone de varios sensores para garantizar el correcto funcionamiento. Aunque pueden variar entre los diferentes modelos se pueden destacar:

- 2 bumpers en la parte frontal que permiten detectar obstáculos que impiden el avance.

- 4 sensores de proximidad en la parte frontal que permiten la detección de obstáculos con anterioridad a que se golpeen los bumpers.

- 2 encoders, uno en cada rueda motriz para detectar cuanto se mueve cada una de ellas

- Un sensor infrarrojo de proximidad en el lateral derecho que permite detectar paredes a escasos milímetros del lateral.

- Un sensor infrarrojo superior para detectar paredes virtuales, la estación de recarga u ordenes con el mando remoto.

Por último, es necesario destacar también que dispone de un puerto serie para su comunicación con el exterior. La configuración por defecto del puerto es a una velocidad de 115200 baudios (aunque para versiones antiguas puede ser diferente), 8 bits de datos, sin paridad y sin control de flujo. Comercialmente existen diferentes dispositivos para comunicación a través de esta interface. Entre ellos se pueden destacar el Roo232, que permite la comunicación a través de una interface RS-232 (por cable), y el RooTooth (Figura 2), que proporciona un puerto serie virtual a través de una comunicación bluetooth (inalámbrica).
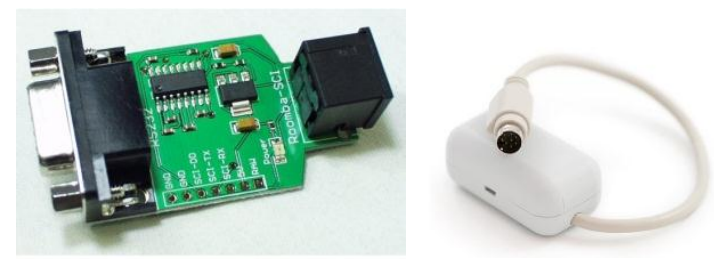

Figura 2: Roo232 y Rootooth.

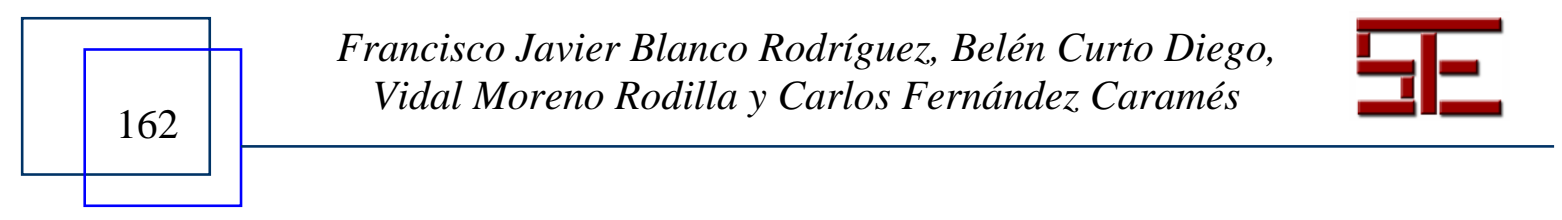




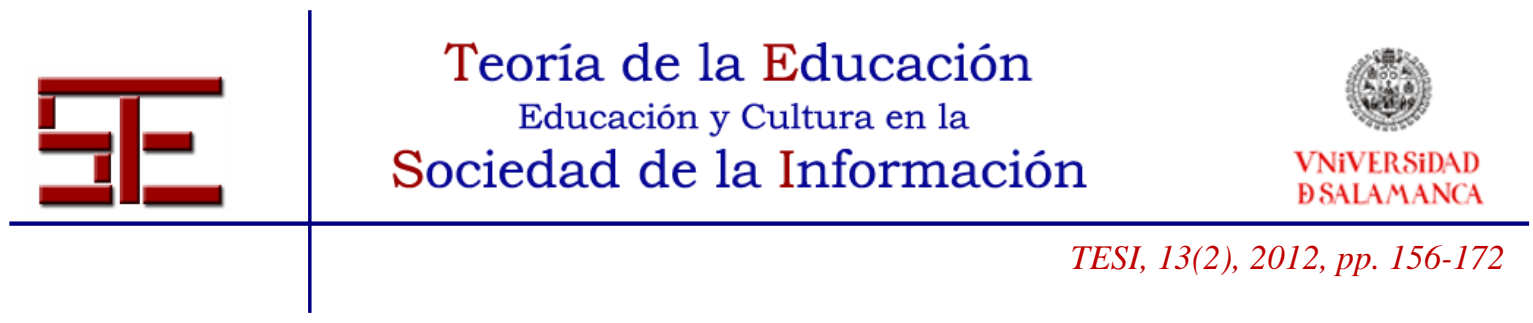

\section{2.- SOFTWARE}

\subsubsection{Firmware del Roomba}

El robot viene provisto con su propio software para desarrollar las tareas propias de limpieza. Este está basado en la arquitectura subsumption [3]. Por lo tanto, su comportamiento es básicamente reactivo realizando movimientos prediseñados hasta que un evento hace que se cambie el tipo de movimiento. Se trata un software cerrado inaccesible completamente a cualquier usuario final.

Sin embargo, iRobot Corporation en diciembre de 2005 con objeto de favorecer el uso de su robot en otras tareas proporcionó la documentación sobre lo que denominó el Serial Command Interface (SCI) a través del puerto serie. En versiones posteriores el conjunto de comandos se vio ampliado pasando a denominarse Roomba Open Interface (ROI). Este protocolo permite la comunicación con el robot tanto para conocer su estado como para controlarlo.

Los comandos permiten, entre otras cosas:

- Activar o desactivar los diferentes motores de limpieza de forma independiente.

- Controlar el movimiento de las ruedas motrices, especificando la velocidad de avance y el radio de giro.

- Obtener el estado de todos los sensores.

\subsubsection{APIs de Programación}

Con objeto de simplificar la comunicación con el robot Roomba y que no sea necesario trabajar con los comandos directamente han aparecido frameworks. Estos son especialmente útiles en este contexto educativo para el cual está planteado el uso de esta plataforma.

Uno de los frameworks que se encuentran disponibles y con una clara orientación a la docencia es LearningRoomba [7]. Proporciona un conjunto de clases en Java integradas dentro del entorno BlueJ [9]. Dentro del proyecto también se proporciona documentación tanto para profesores como para estudiantes orientada a sesiones de clases prácticas. Sin embargo, BlueJ, que proporciona un entorno de programación muy

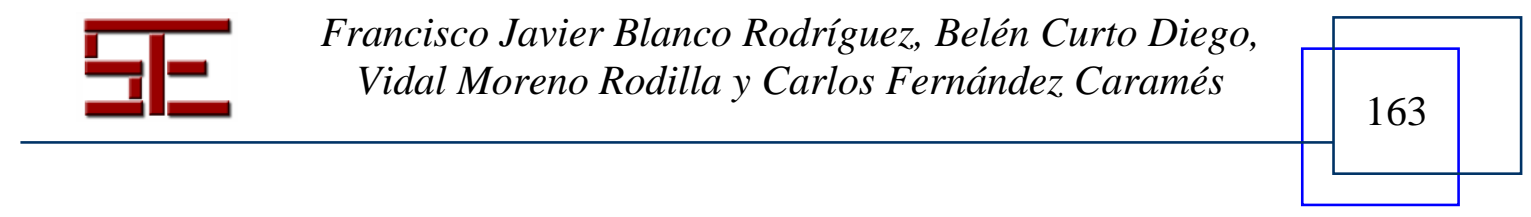




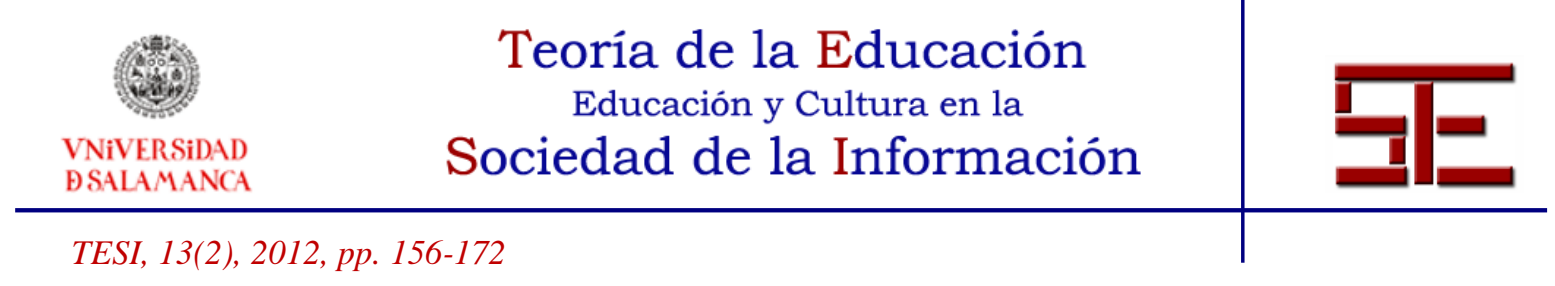

sencillo, puede resultar demasiado limitado para alumnos en los últimos cursos de un Grado en Ingeniería Informática.

Otro framework considerado ha sido roomba-term, desarrollado por Kevin Gabbert bajo licencia OSL 3.0. Está desarrollado en el lenguaje C\# como un proyecto de Microsoft Visual Studio. El principal inconveniente radica en las dificultades en la portabilidad.

El último framework considerado ha sido RoombaComm, desarrollado por Tod E. Kurt y Paul Bouchier bajo licencia GNU LGPL. Está completamente desarrollado en Java proporcionando un conjunto de clases agrupadas en un paquete jar. Al estar desarrollado en Java tiene un carácter multiplataforma pudiendo utilizarse en Windows, Linux o Mac OS X. La programación por tanto se puede realizar bajo cualquier IDE de desarrollo que soporte Java lo cual para los alumnos de los últimos cursos del Grado no supondrá ningún esfuerzo añadido. Otro valor añadido es que uno de los desarrolladores es el autor del libro Hacking Roomba: ExtremeTech [10] donde se hace una amplia explicación del hardware del robot y programación utilizando este framework.

Así de este amplio abanico de posibilidades se ha optado por RoombaComm por ser lo suficientemente sencilla y flexible para una materia en los últimos cursos de Ingeniería Informática. Así, dada la naturaleza de los estudiantes, la familiarización con una biblioteca de Java se realiza rápidamente pudiendo pasar casi inmediatamente experimentar con el robot enviándole comandos.

\section{METODOLOGÍA}

La metodología utilizada para la docencia de la práctica de esta materia busca la participación activa del alumnado acorde a la filosofía del Espacio Europeo de Educación Superior. Ya los contenidos tratados en las clases teóricas tendrán un papel motivador, tratando los aspectos más interesantes y actuales de la robótica. Pero en la parte práctica se pretende ir más lejos todavía logrando que los estudiantes se planeen su formación como un reto personal.

Así, se pretende conseguir que los estudiantes no se limiten a seguir las indicaciones de los correspondientes guiones de prácticas de forma pasiva, y sin tener que tomar apenas decisiones. Por el contrario, deberán ser ellos los que tengan que decidir qué pasos seguir en cada momento. Para ello, se plantearán una serie de proyectos, en grado de

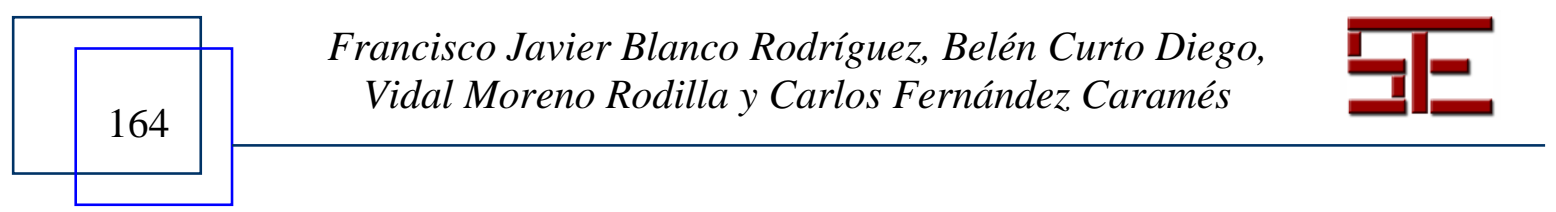




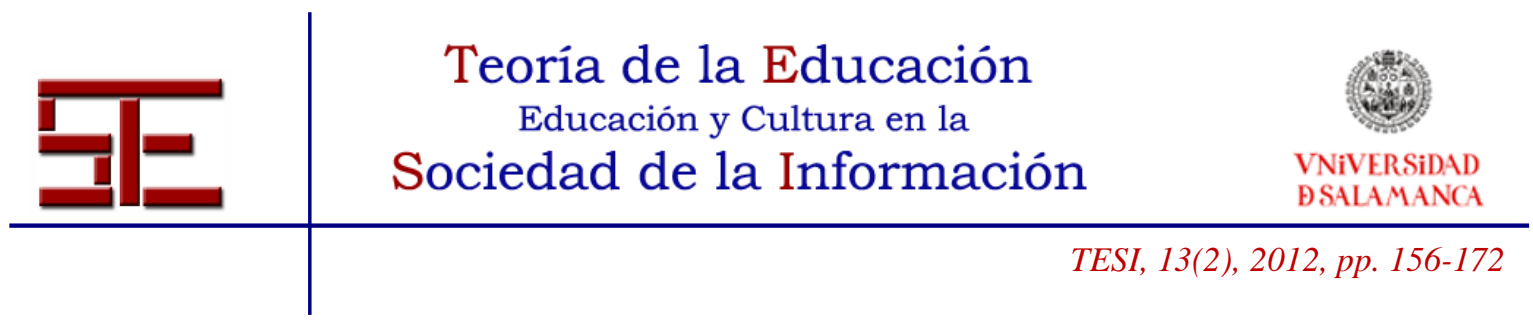

dificultad creciente, que deberán realizar de forma independiente cada uno de los grupos de trabajo.

Estos proyectos, lejos de enunciarse mediante un texto escrito, se definirán mediante videos que muestren a los robots realizando diferentes acciones y comportamientos inteligentes. De este modo, un primer paso en el proceso del aprendizaje vendrá dado por el análisis necesario y la relación entre lo visto y los contenidos teóricos vistos previamente.

Así, antes de empezar cada proyecto se organizará un seminario en el que mediante una lluvia de ideas los estudiantes deben realizar análisis del proyecto propuesto. Se fomentará la participación en el debate, teniendo en cuenta esta en la evaluación de la asignatura. Como resultado de la sesión cada grupo de trabajo debe extraer que está haciendo el robot y qué sensores es necesario utilizar para conseguirlo.

Posteriormente en cada grupo de trabajo deben debatir y proponer un algoritmo que consiga reproducir los movimientos del robot del mismo modo que vieron en el video.

Posteriormente deberán implementar el algoritmo propuesto y probar su funcionamiento. En caso de que con los pasos realizados previamente no sean capaces de obtener una solución aceptable deberán volver a realizar el análisis del material audiovisual proporcionado para proponer la utilización de otros sensores $\mathrm{u}$ otro algoritmo.

Las conclusiones obtenidas en el seminario como el debate de grupo deberán adjuntarlas a modo de informe junto con el proyecto final, analizando también el grado de consecución de los objetivos previstos.

Con objeto de buscar un mayor entusiasmo se planteará un procedimiento de evaluación en el que serán los propios alumnos quienes fijen un porcentaje de su nota final. Así, al finalizar el curso se realizará una sesión en la que se presentará cada proyecto por equipos de trabajo.

Posteriormente, se realizan dos encuestas anónimas. La primera de ellas estará destinada a medir la calidad del proyecto realizado. Así, consistirá en una votación individual de acuerdo al resultado obtenido y originalidad en la propuesta. La segunda estará enfocada a valorar el grado de participación de cada uno de los miembros del grupo. En

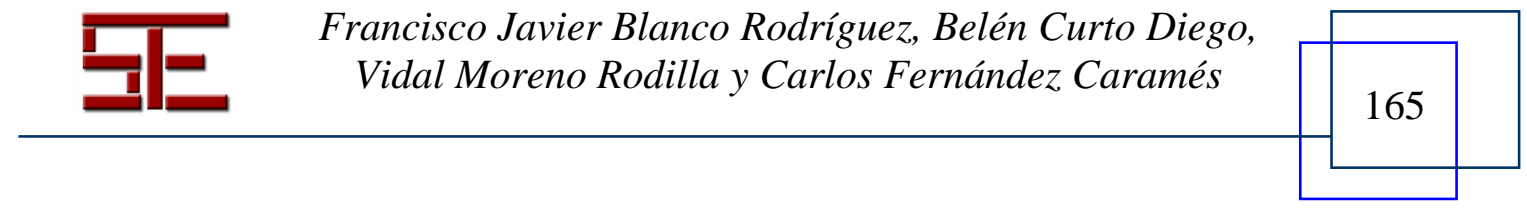




\begin{tabular}{c} 
Teoría de la Educación \\
Educación y Cultura en la \\
$\begin{array}{c}\text { VNiVERSIDAD } \\
\text { BSALAMANCA }\end{array}$ \\
\hline TESI, 13(2), 2012, pp. 156-172
\end{tabular}

esta cada alumno deberá especificar el grado de trabajo y aportaciones del resto de miembros del grupo al que pertenece.

Así los proyectos propuestos serán los siguientes:

\subsection{Exploración de un entorno con movimientos simples}

Este proyecto consiste en que el robot se mueva por el entorno intentando llegar a todos los lugares accesibles. Para esto, el robot se moverá en línea recta hasta que alcance algún obstáculo o pared. En ese momento el robot retrocederá ligeramente para separarse de la pared y girará un ángulo aleatorio en el sentido contrario al que se ha encontrado el obstáculo.

Con este comportamiento se puede comprobar que es posible explorar una habitación y en el caso de que se trate de un pasillo avanzar en zig-zag.

En la figura 3 se muestra la secuencia de movimientos que percibirían los alumnos.

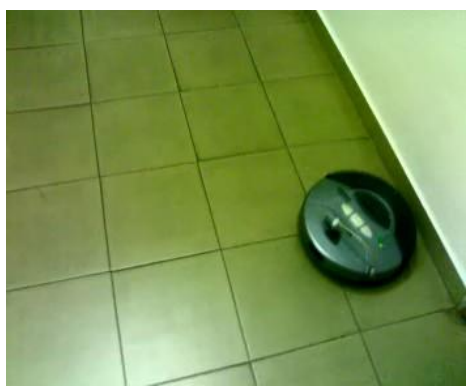

a)

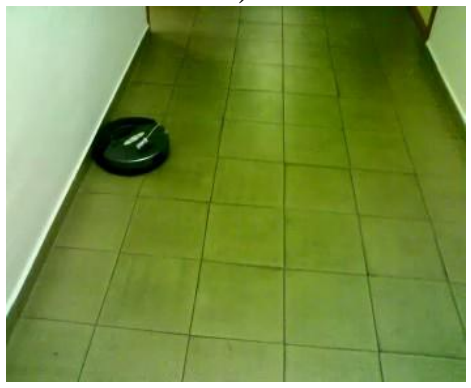

d)

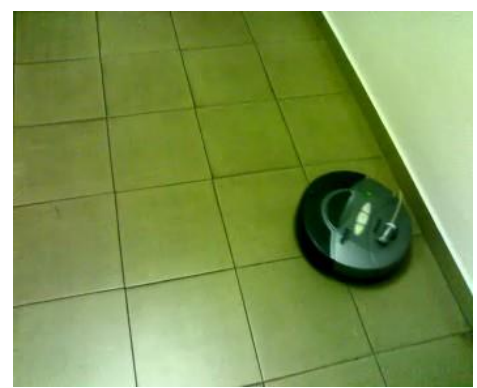

b)

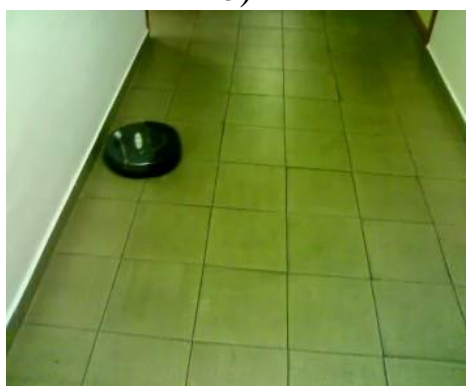

e)

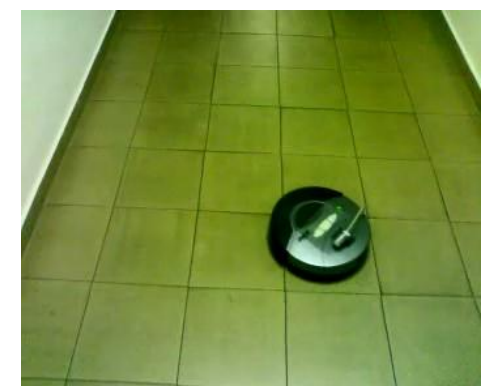

c)

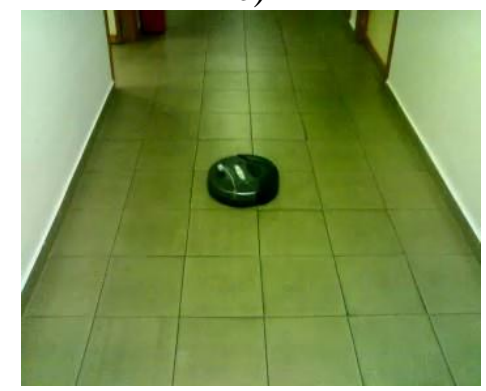

f)

Figura 3: Secuencia de movimientos del robot explorando.

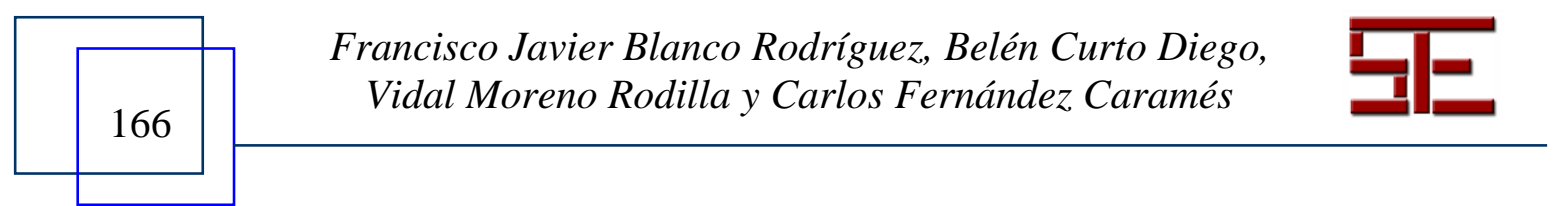




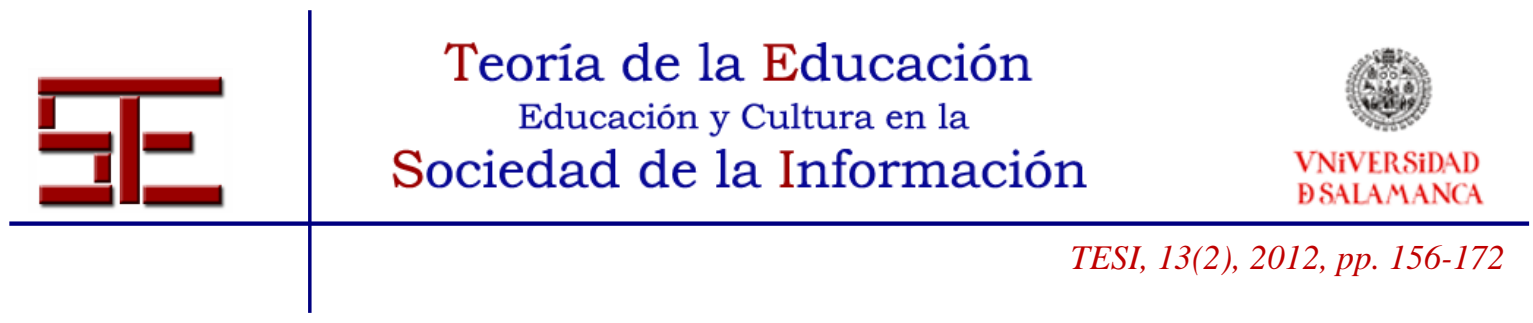

El objetivo de este proyecto es que los alumnos se familiaricen con la programación del robot realizando movimientos muy sencillos y utilicen unos sensores tan sencillos como son los bumpers.

\subsection{Movimiento a lo largo de una pared}

En este proyecto el robot debe moverse hasta que encuentre una pared. Una vez alcanzada debe avanzar pegado a la pared dejándola a su derecha, como se muestra en la Figura 4. Este comportamiento es similar a uno de los que vienen incorporados en el firmware del robot por lo que los alumnos pueden comparar los resultados que obtienen con los que ya realizaba el robot.

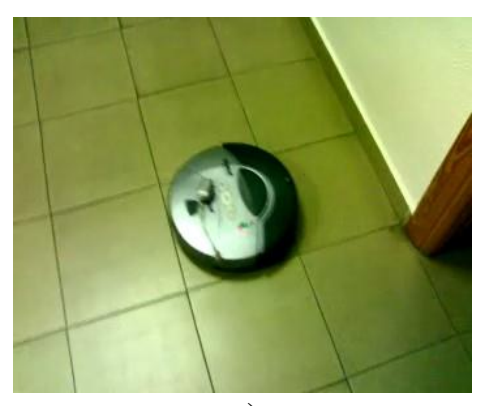

a)

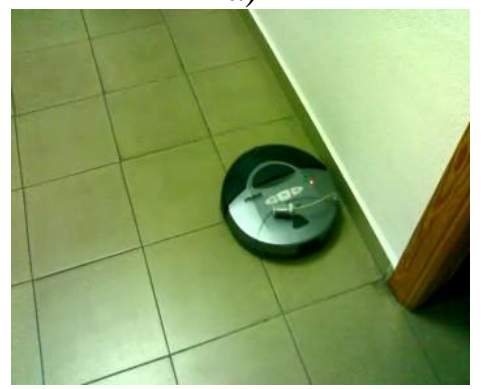

d)

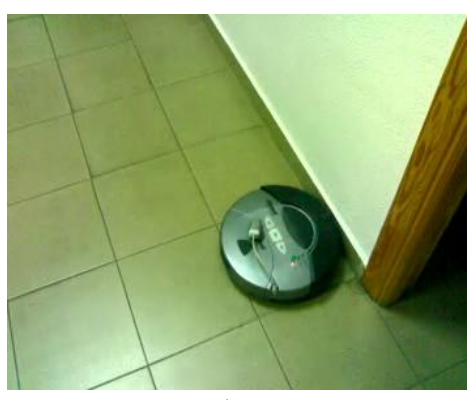

b)

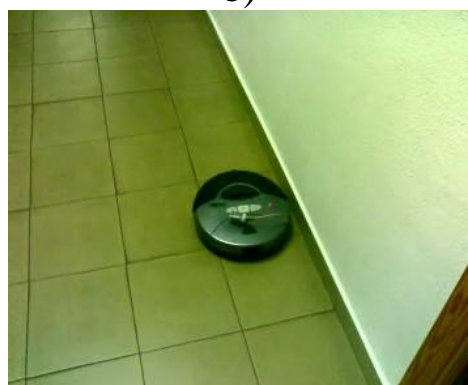

e)

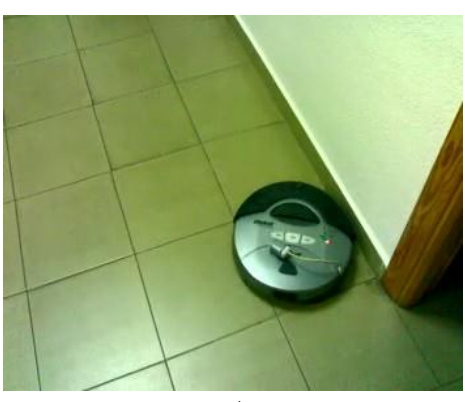

c)

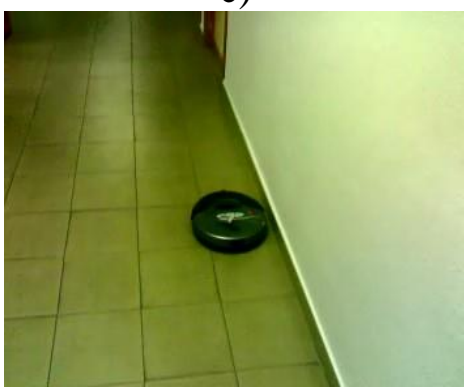

f)

Figura 4: Secuencia de movimientos del robot siguiendo la pared.

El objetivo de este proyecto es que se comiencen a realizar movimientos más complejos que en el proyecto anterior, pudiéndose mover el robot con trayectorias curvas y no solamente rectas. Además deberán utilizar un nuevo sensor que permite detectar una pared en la parte derecha del robot cuando se encuentra a menos de $1 \mathrm{~cm}$.

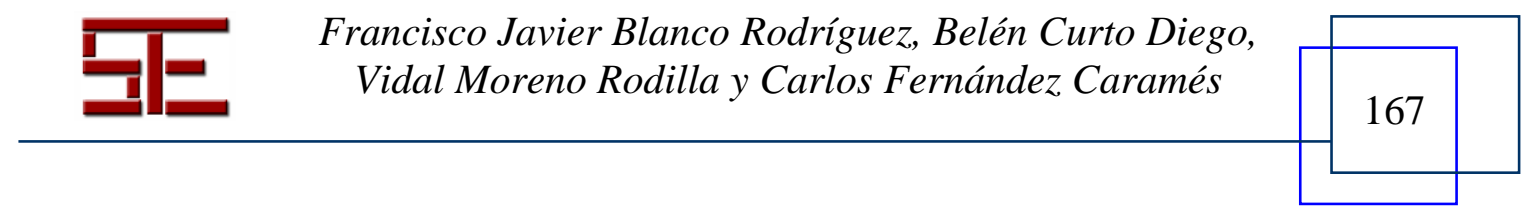




\begin{tabular}{cc} 
Teoria de la Educación \\
Educación y Cultura en la \\
$\begin{array}{c}\text { VNiVERSIDAD } \\
\text { BSALAMANCA }\end{array}$ \\
\hline TESI, 13(2), 2012, pp. 156-172
\end{tabular}

\subsection{Sumo}

En este proyecto los estudiantes deberán conseguir que el robot reproduzca el comportamiento de los robots en los concursos de sumo. Para ello el robot se encontrará en un ring delimitado por paredes virtuales. Este debe moverse dentro del ring sin salirse hasta que detecte la colisión con cualquier objeto, momento en el que deberá intentar empujarlo fuera.

Con este proyecto se busca que el alumnado además de continuar utilizando nuevos sensores afiance las habilidades adquiridas con los proyectos anteriores trabajando con un proyecto mucho más atractivo que además permite hacer competiciones entre los equipos de trabajo.

\subsection{Movimiento siguiendo una trayectoria}

Los estudiantes al realizar los proyectos anteriores se habrán dado cuenta de que hasta ahora solamente han realizado comportamientos reactivos, determinando qué acción realizar según el estado de los sensores.

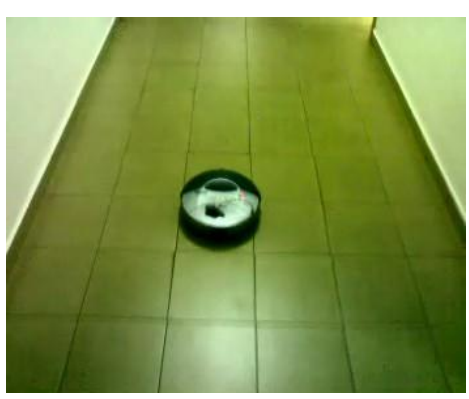

a)

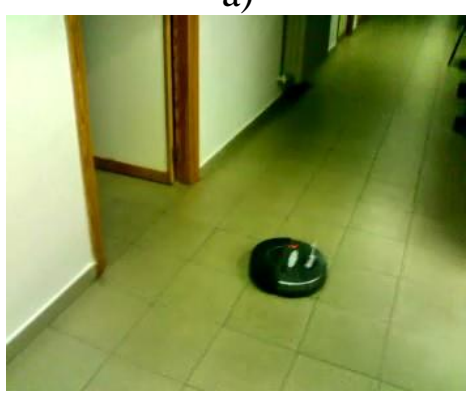

d)

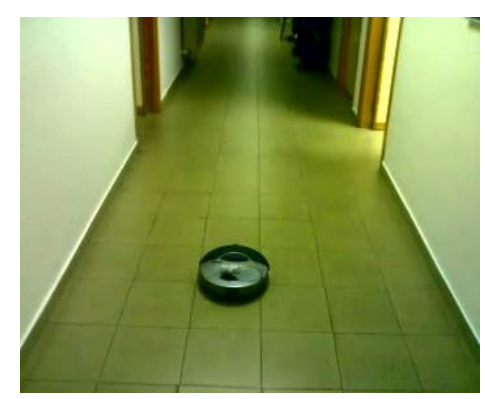

b)

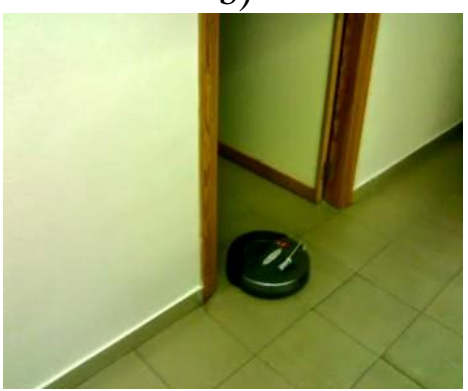

e)

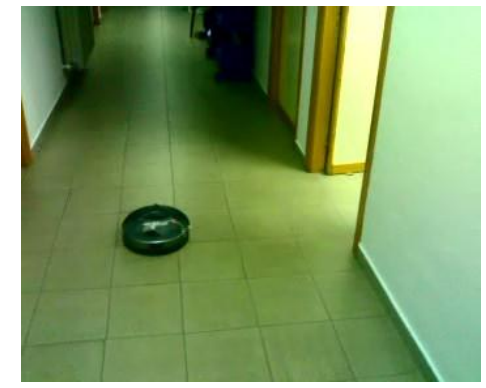

c)

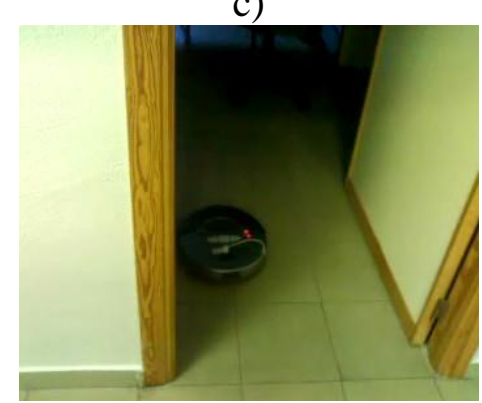

f)

Figura 5: Secuencia de movimientos del robot siguiendo una trayectoria.

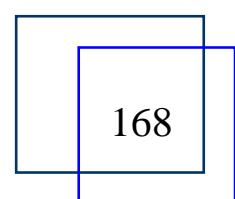

Francisco Javier Blanco Rodríguez, Belén Curto Diego, Vidal Moreno Rodilla y Carlos Fernández Caramés

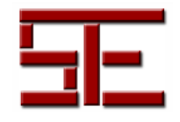




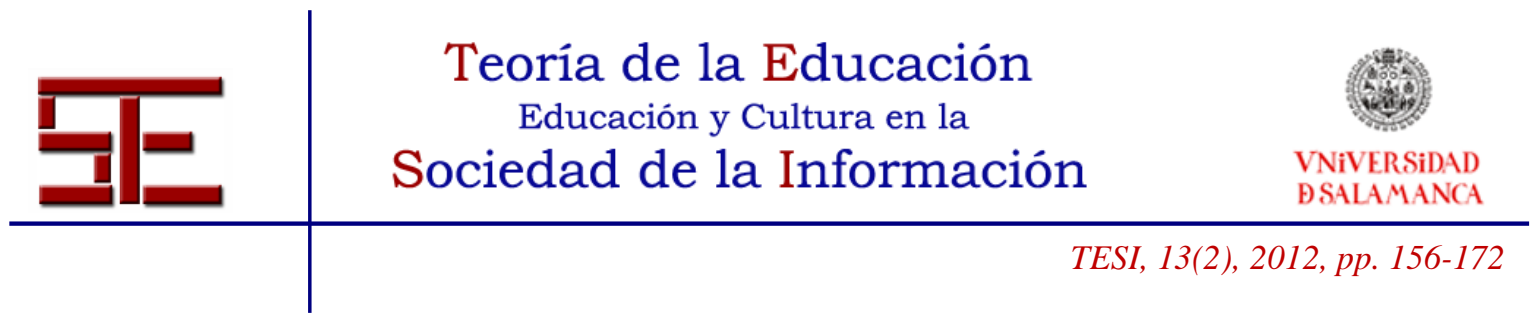

En este proyecto en el video observarán a un robot realizando una trayectoria compleja y precisa como la que se puede observar en la Figura 5. En ella el robot se mueve por el medio de un pasillo y posteriormente entra por una puerta.

Con este proyecto los alumnos deberán darse cuenta de la necesidad de contar con una realimentación de los movimientos realizados por el robot a través de los encoders acoplados a los motores de las ruedas. De tal modo que si pretendemos realizar un control deliberativo podamos conocer en qué posición se encuentra el robot en cada momento.

\subsection{Estimación de los errores en la odometría}

Partiendo de los resultados obtenidos en el proyecto anterior, se propondrá al alumnado que proporcionen al robot una trayectoria que consista en realizar varias veces el cuadrado unidireccional propuesto por Borenstein [2].

Aunque se trata de un proyecto sencillo, les permitirá comprobar que la posición estimada por medio de los encoders es imprecisa y que además aumenta con la distancia recorrida. Así, este será un buen momento para incidir en que un robot móvil debe contar con algún sistema de posicionamiento absoluto para corregir los errores de posición.

\subsection{Planificación local}

Llegados a este punto, para finalizar, se propondrá a los alumnos que utilizando todo lo que han aprendido y desarrollado anteriormente implemente alguno de los algoritmos de bug propuestos por Lumelsky. Así, el robot deberá ir desde el punto donde se encuentre hasta su destino y en el momento que detecte algún obstáculo con los bumpers rodearlo hasta que pueda seguir en línea recta hasta su destino.

Un ejemplo de las secuencias del video que se proporciona a los alumnos para que obtengan las características de los comportamientos que deben reproducir se muestra en la Figura 6.

Este proyecto servirá de colofón de la asignatura de modo que los alumnos deban enfrentarse a un proyecto complejo que aúne todos los proyectos anteriores y de este

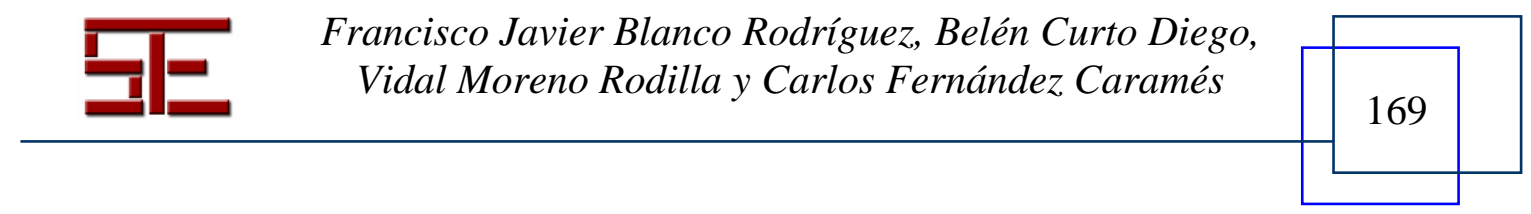




\begin{tabular}{c} 
Teoria de la Educación \\
Educación y Cultura en la \\
$\begin{array}{c}\text { VNiVERSIDAD } \\
\text { BSALAMANCA }\end{array}$ \\
\hline TESI, 13(2), 2012, pp. 156-172
\end{tabular}

modo puedan demostrar todos los conocimientos y habilidades adquiridos en la asignatura.

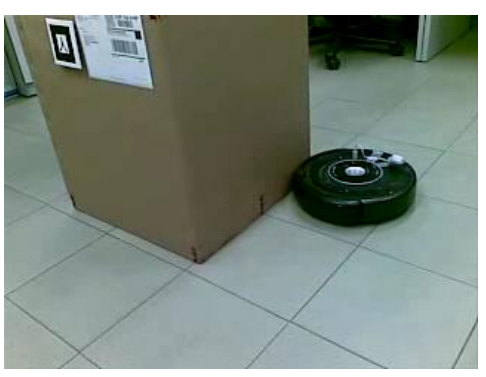

a)

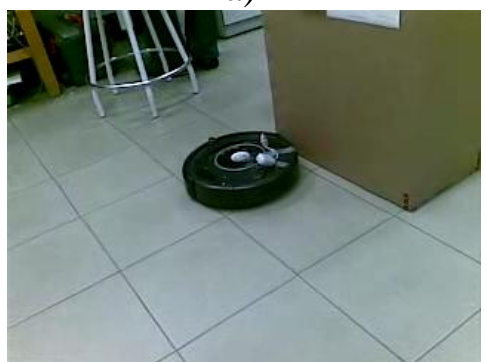

d)

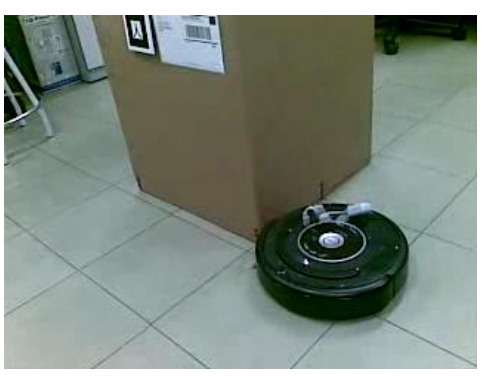

b)

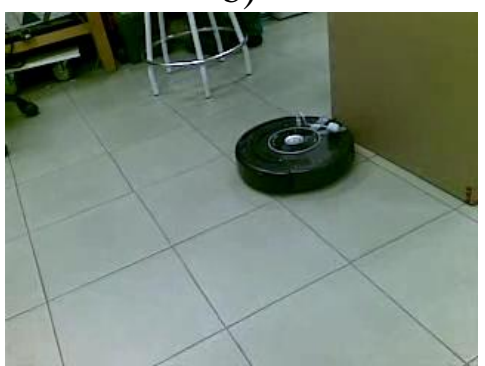

e)

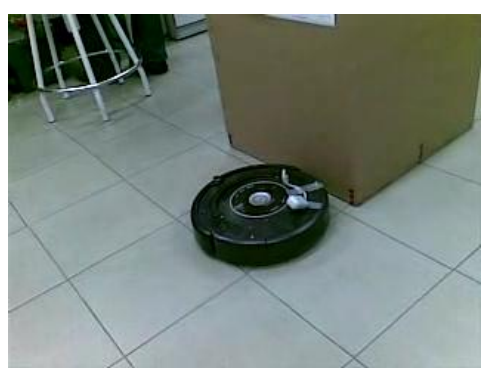

c)

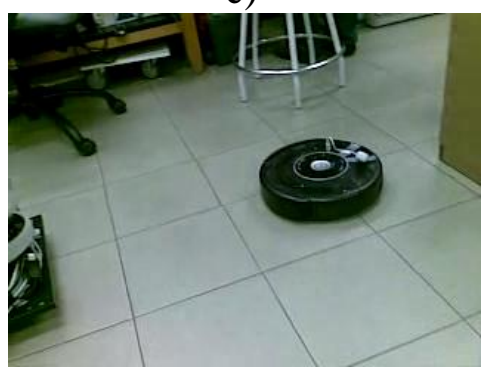

f)

Figura 6: Secuencia de movimientos del robot rodeando un obstáculo.

\section{CONCLUSIONES}

El robot Roomba se ha propuesto como plataforma para la enseñanza práctica en el campo de la robótica en el Grado en Ingeniería Informática. Junto con su bajo coste, una característica importante que ha sido fundamental en su elección es la posibilidad de envío de órdenes desde un ordenador remoto mediante frameworks en lenguajes de alto nivel, por lo que el aprendizaje a los estudiantes de este Grado les puede resultar muy atractivo y servir para afianzar sus conocimientos de programación. También, los estudiantes se podrán centrar en los aspectos propios de la materia que en los entornos de desarrollo o lenguajes de programación específicos para un robot.

Otra característica que puede motivar especialmente a los alumnos es que no solamente no se trata de un robot simulado, por lo que se presentan problemas que no aparecen en simulación, sino que además sea un robot comercial con características autónomas. Esto

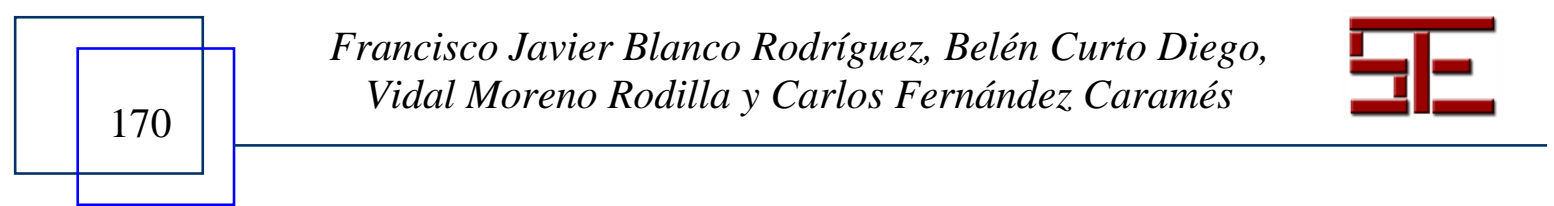




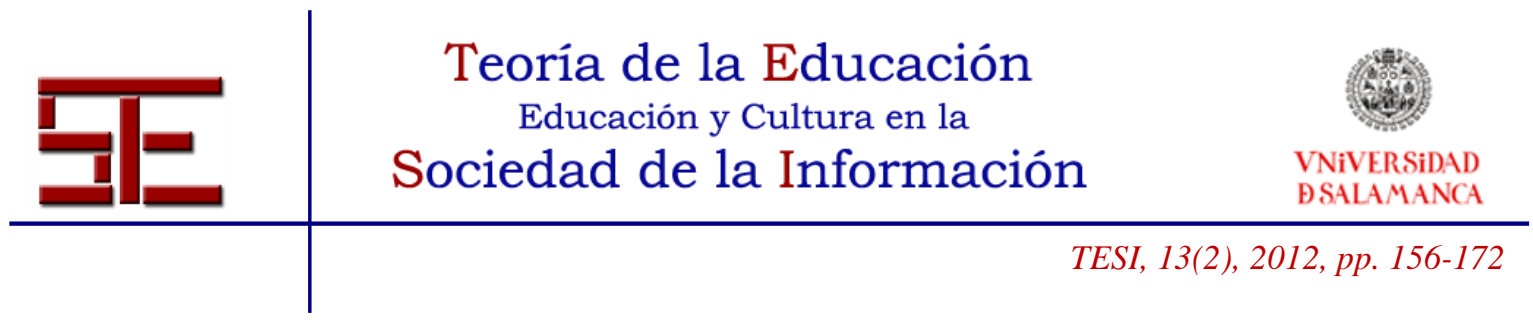

hace que las prácticas se conviertan en retos intentando simular e incluso mejorar los comportamientos con los que viene de fábrica.

Se propone también una metodología docente basada en videos proyectos con objeto de conseguir una mayor participación activa de los alumnos, tal y como debe ser en una materia con una alta carga práctica. Así, el alumnado dejará de ser un mero receptor de conocimiento para pasar a ser una pieza fundamental del proceso de enseñazaaprendizage teniendo que asimilarlos y desarrollar las habilidades prácticas.

Para concluir, en este artículo se han propuesto una serie de proyectos con un grado de dificultad creciente. Así se culminará con un proyecto complejo en el que se aúne todo lo aprendido.

\section{BIBLIOGRAFÍA}

Arkin, R. C. (1998). Behavior-based Robotics. Cambridge, MA: MIT Press.

Borenstein, J., Feng, L. (1996). Measurement and Correction of Systematic Odometry Errors in Mobile Robots. Transactions on Robotics and Automation, 12, 869-880.

Brooks, R. A. (1991). Intelligence Without Representation. Artificial Intelligence 47, pp. 139-159.

Daisy Fan, K.-Y., Kathryn Conway, D. (2011, octubre). Using the Matlab-based iRobot create simulator to engage introductory computer programming students in program development and observing computational errors. 2011 Frontiers in Education Conference, Rapid City, SD, USA.

Dickinson, B.; Jenkins, O.; Moseley, M.; Bloom, D.; and Hartmann, D. (2007, marzo). Roomba pac-man: Teaching autonomous robotics through embodied gaming. AAAI Symposium on Robot and Robot Venues: Resources for AI Education. Stanford, CA, USA.

González de Rivera, G., Ribalda, R., Garrido, J. (2006). Diseño de un Laboratorio de Robótica Autónoma. Universidad Autónoma de Madrid. Tecnologías Aplicadas a la Enseñanza de la Electrónica.

Housten, D. T. (2008). Robotics Toolkit for Pre-College Engineering Education. Master Thesis. Computer Science Department, Drexel University, Philadelphia, PA, USA.

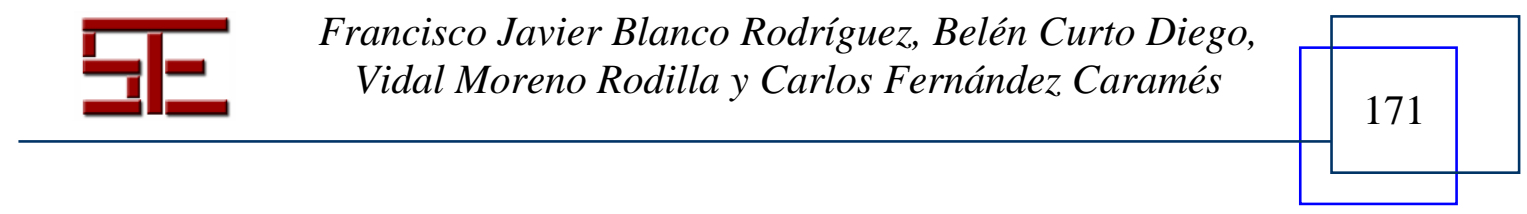




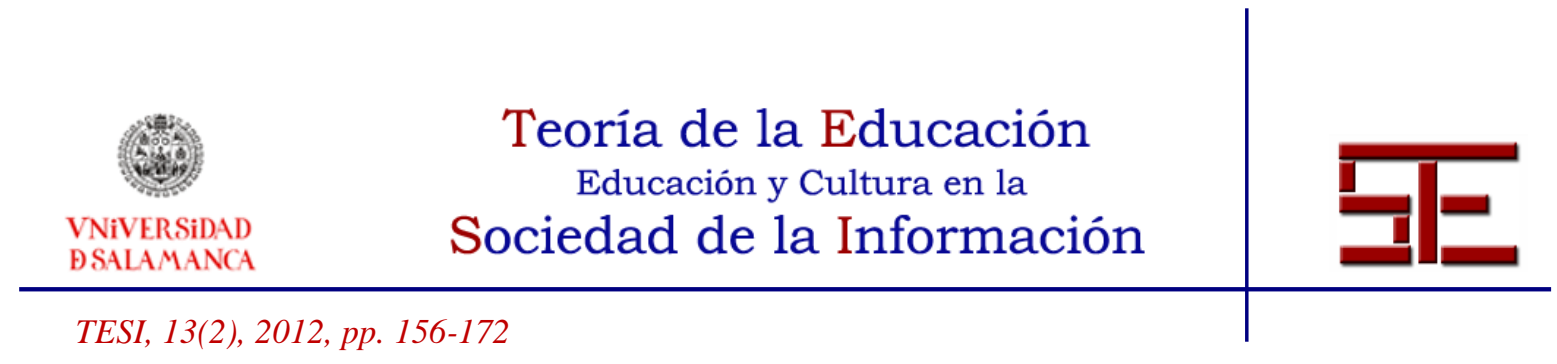

Isaacs, J. T., Klein, D. J., Hespanha, J. P. (2011, septiembre). A Guided Internship For High School Students Using iRobot Create. 18th IFAC World Congress, Milano, Italia.

Kölling, M., Quig, B., Patterson, A., y Rosenberg, J. (2003). The BlueJ System and its Pedagogy. Computer Science Education, 13 (4), 249-268.

Kurt, T. E. (2006). Hacking Roomba: ExtremeTech. New York, NY: John Wiley \& Sons, Inc.

Leitão, P., Gonçalves, J., Barbosa, J., (2005). Learning Mobile Robotics using LEGO Mindstorms. En 9th Congresso Hispano Luso de Engenharia Electrotécnica.

Menegatti, E., Moro, M. (2010, noviembre). Educational Robotics from high-school to Master of Science. Intl. Conf. on Simulating, Modeling and Programming for Autonomous Robots.

Miller, D. P. (2004, marzo). Using Robotics to teach computer Programming \& AI Concepts to Engineering Students. AAAI Spring Symposium on Accessible Handson Artificial Intelligence and Robotics Education. Stanford, CA, USA.

Tribelhorn, B. \& Dodds, Z. (2007). Evaluating the Roomba: A low-cost, ubiquitous platform for robotics research and education. En IEEE International Conference on Robotics and Automation, 1393-1399.

Para citar el presente artículo puede utilizar la siguiente referencia:

Blanco Rodríguez, F. J., Curto Diego, B., Moreno Rodilla, V. y Fernández Caramés, C. (2012). Propuesta docente para las prácticas de róbotica en el Grado de Ingeniería Informática. Revista Teoría de la Educación: Educación y Cultura en la Sociedad de la Información. 13(2), 156-172 [Fecha de consulta: dd/mm/aaaa].

http://campus.usal.es/ revistas_trabajo/index.php/revistatesi/article/view/9020/9264

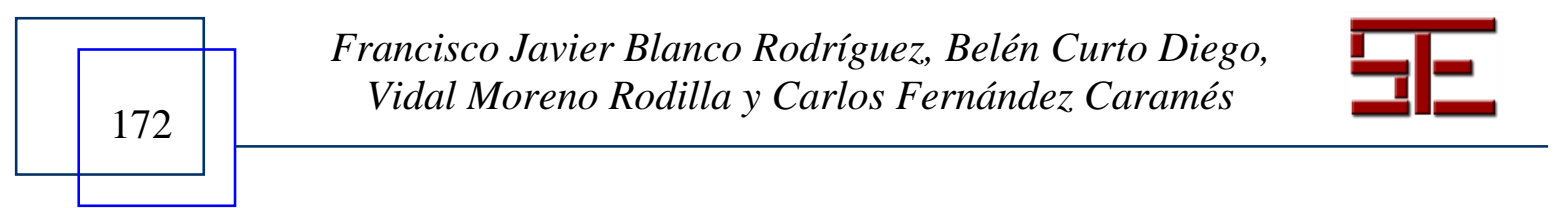

